\title{
Stress field in deformed polycrystals at the micron scale
}

\author{
O. Castelnau ${ }^{1, \mathrm{a}}$, M. Bornert ${ }^{2}$, O. Robach ${ }^{3}$, J.S. Micha ${ }^{3}$, O. Ulrich ${ }^{3}$, R. Chiron ${ }^{1}$, C. Le Bourlot ${ }^{1}$ \\ ${ }^{1}$ LPMTM, CNRS, Université Paris 13, 93430 Villetaneuse, France \\ ${ }^{2}$ Unité de Recherche NAVIER, École des Ponts ParisTech, 77455 Marne-la-Vallee, France \\ ${ }^{3}$ ESRF, 38043 Grenoble, France
}

Theoretical, numerical, and experimental micromechanical approaches have been the subject of significant improvements during the last decade, from the scale of the lattice defect (nanometre) up to the scale of the polycrystal (millimetre). When plastic deformations take place in polycrystals, strong and systematic strain heterogeneities build up between neighbouring grains but also inside individual grains. These heterogeneities have to be (or should be) considered for many issues, e.g. onset of microplasticity, crack propagation, phase transformation, activation of twinning, or simply the prediction of the effective behaviour of the material. From the experimental point of view, Digital Image Correlation (DIC) techniques adapted to images obtained by Scanning Electron Microscopy generally reveal strain localization bands the extent of which is typically 5 to 10 grain sizes. The prediction of such behaviour with theoretical or numerical scale transition models is however most of the time severely limited by the poor knowledge of the local behaviour at the grain or at an even finer scale. Furthermore, although significant progress could be reached by several research groups as for the characterization of the displacement/strain fields by DIC, one has to recognize that similar developments for the characterization of the stress field are far less spectacular. This comes probably from the high accuracy that has to be obtained on elastic strain measurements to get reliable stress estimation. Combining this difficulty with a micrometric spatial resolution as needed for standard micromechanical problems is not simple.

The present work is a contribution to the development of an X-ray Laue microdiffraction technique, which is well adapted for the characterization of the stress field in deformed polycrystals at the micron scale. The technique consists in scanning the specimen in front of a focussed white Xray beam with micrometric cross section. The shape of the resulting Laue diffraction patterns reveals many features relative to the specimen deformation and among them the elastic strain of interest here. X-ray Laue microdiffraction setup has been developed and installed on few synchrotron facilities such as ALS-Berkeley and APS-Argonne in the USA, and ESRF in France. However, although the technique is very promising, only very few quantitative results have been published so far, since poorly appropriate data treatment method and software has been proposed so far. We aim at developing and applying an adapted procedure by putting together different experimental and image processing techniques, in particular Laue microdiffraction as available at beamline BM32 of the ESRF, and Digital Image Correlation (DIC) for the precise evaluation of the evolution of the Laue pattern in the form of an apparent heterogeneous displacement related to the lattice distorsion (figure 1). Particular attention is placed on the careful investigation of experimental and image processing uncertainties in order to evaluate precisely the experimental errors on stress evaluation.

\footnotetext{
a Email: oc@1pmtm.univ-paris13.fr
} 
We will show results obtained during in situ tensile tests on a polycrystalline specimen of tungsten (W). The specimen was deformed elastically, i.e. for applied macroscopic stresses far below the yield stress. In doing so, owing to the local elastic isotropy of W grains, a uniform stress field is generated throughout the specimen. We make use of this property to provide a robust estimation of the uncertainties obtained on the stress field.
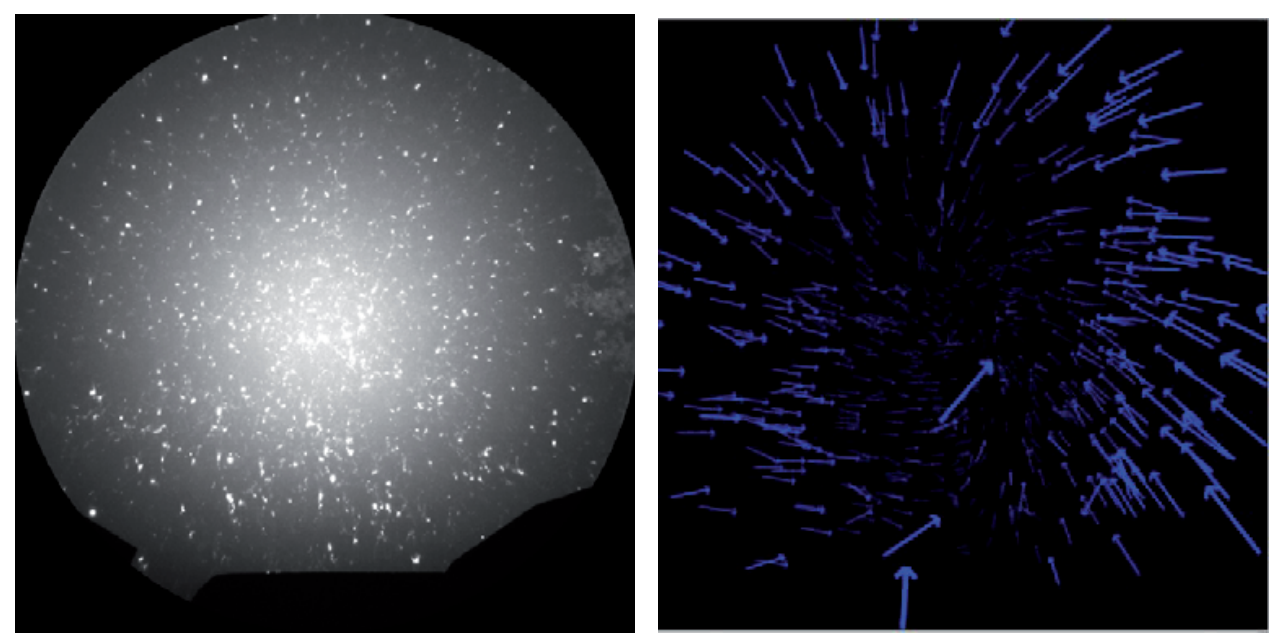

Fig. 1. (left) Typical Laue pattern obtained on the tungsten polycrystal during in situ tensile test at beamline BM32 (ESRF). The X-ray beam cross section was $~ 1 \times 1 \mu \mathrm{m}$. (right) Relative displacement of Laue spots for a macroscopic stress increment of $500 \mathrm{MPa}$, revealing the local (intragranular) elastic strain at the micron scale. Length of arrows is multiplied by a factor 50 . 